\title{
Weiner Polynomials to Generalize the Distance of Some Composite Graphs from Special Graphs
}

Ali A. Ali

$$
\begin{gathered}
\text { aliazizali1933@yahoo.com ahmedgraph@uomosul.edu.iq } \\
\text { College of Computer Sciences and Mathematics } \\
\text { University of Mosul, Iraq }
\end{gathered}
$$

Accepted on: 24/12/2006

\begin{abstract}
It is not easy to find the Wiener polynomials for generalized distance of compound graphs constructed in the form $G_{1} \bullet G_{2}$ and $G_{1}: G_{2}$ for any two disjoint connected graphs $\mathrm{G}_{1}$ and $\mathrm{G}_{2}$. Therefore, in this paper, we obtain Wiener polynomials for generalized distance of $G_{1} \bullet G_{2}$ and $G_{1}: G_{2}$ when $\mathrm{G}_{1}$ and $\mathrm{G}_{2}$ are special graphs such as complete graphs, and stars. The Wiener index of each such graph with respect to the generalized distance is also obtained in this paper.

The Wiener polynomial for the generalized distance of the join of any two disjoint connected graphs is also obtained in this paper.

Keywords : n-distance, Wiener polynomials, Composite Graphs.

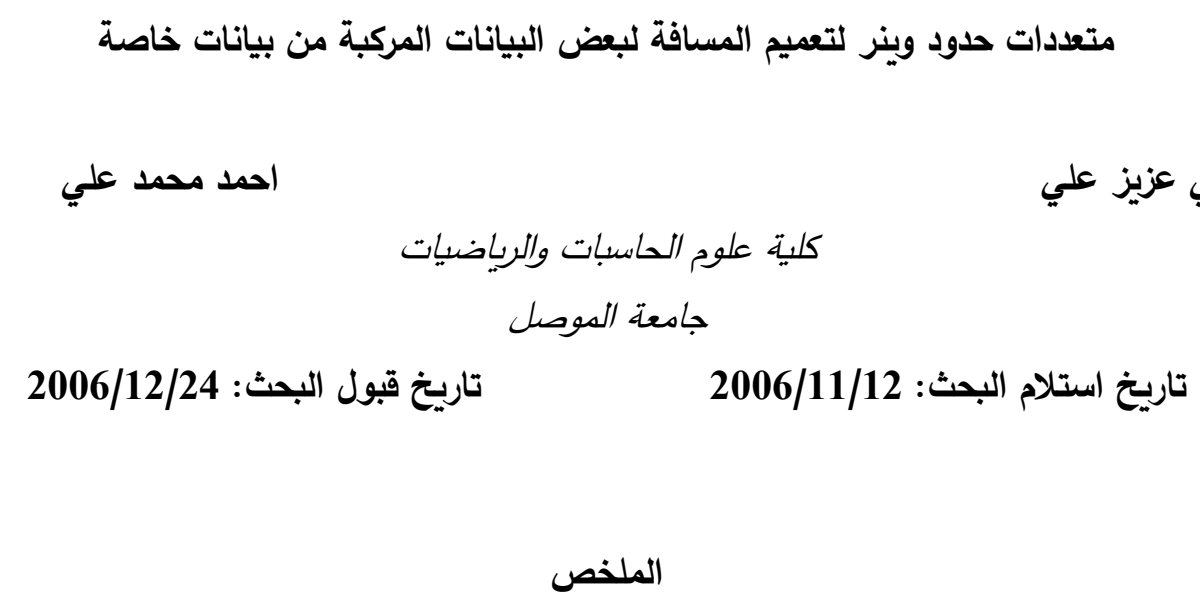


عالجنا هذه المشكلة عندما يكون البيانين G و G $\mathrm{G}_{2}$ خاصين، مثل البيانات التامة ، وبيانات النجمة. ولقد أوجدنا دليل وينر بالنسبة الى تعميم المسافة هذه لكل من البيانات المذكورة.

ولقد ذكرنا في الفقرة الثانية من هذا البحث صيغة لإيجاد متعددة حدود وينر لتعميم المسافة لبيان مركب من اتصال بيانين منفصلين. الكلمات المفتاحية : المسافة -n ، متعددة حدود وينر ، تركيب البيانات.

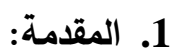

قام العديد من الباحثين بتعميم المسافة بين مجموعتين من الرؤوس [2]،[3] ، والتي

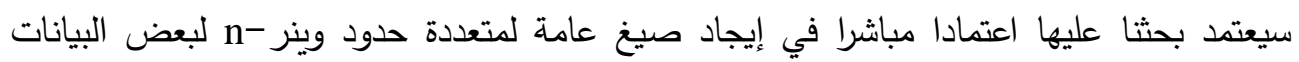

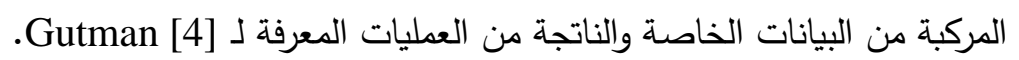

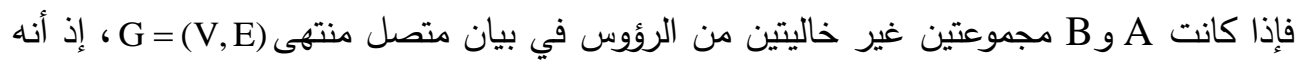

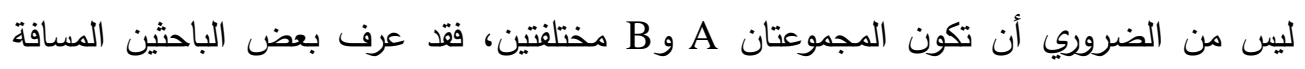

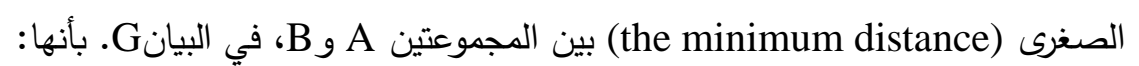
$d_{\min }(A, B)=\min \{d(a, b): a \in A, b \in B\}$

تعريف (1.1): ليكن G بيانا متصلا برتبة p، ولتكن S مجموعة-(n-1) من رؤوس G م ، إذ

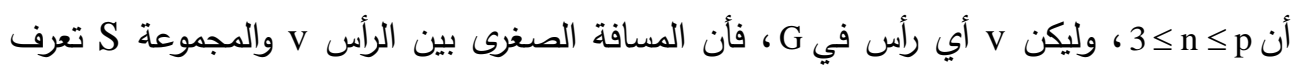
كالأتي: $\left.d_{n}(v, S)=\min \{d(v, u): u \in S\}\right)$

واضح أن

$\mathrm{d}_{\mathrm{n}}(\mathrm{v}, \mathrm{S})=0$; when $\mathrm{v} \in \mathrm{S}$

$d_{n}(v, S) \geq 1$; when $\quad v \notin S$

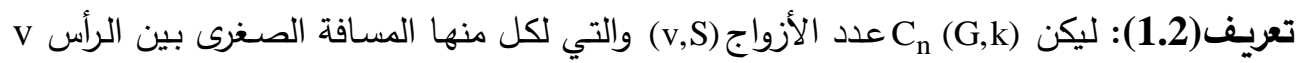

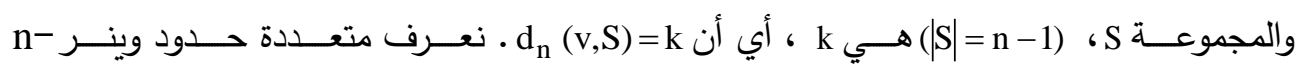
للبيان بأنها متعددة الحدود والتي يرمز لها Wh $\mathrm{W}_{\mathrm{n}}(\mathrm{G} ; \mathrm{x})=\sum_{\mathrm{k}=0}^{\delta_{\mathrm{n}}} \mathrm{C}_{\mathrm{n}}(\mathrm{G}, \mathrm{k}) \mathrm{x}^{\mathrm{k}}$ 
إذ أن d هو قطر لهذا النوع من المسافة للبيان G، أي أن

$$
\delta_{\mathrm{n}}=\max \{\mathrm{d}(\mathrm{v}, \mathrm{S}): \mathrm{v} \in \mathrm{V}, \mathrm{S} \subseteq \mathrm{V},|\mathrm{S}|=\mathrm{n}-1\}
$$

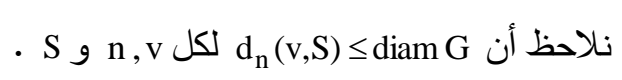

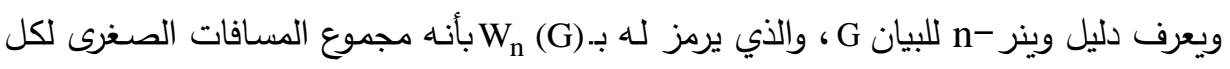

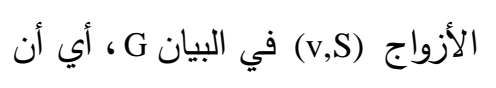

$$
\mathrm{W}_{\mathrm{n}}(\mathrm{G})=\sum_{\substack{(\mathrm{v}, \mathrm{S}) \\ \mathrm{v} \in \mathrm{V}, \mathrm{S} \subseteq \mathrm{V}}} \mathrm{d}_{\mathrm{n}}(\mathrm{v}, \mathrm{S})
$$

$$
\text { | }|S|=n-1)
$$

وكذلك يمكن التعبير عن دليل وينر - بأنه مشتقة متعددة حدود وينر n- بالنسبة الى xندما x=1 كئ

$\mathrm{W}_{\mathrm{n}}(\mathrm{G})=\left.\mathrm{W}_{\mathrm{n}}^{\prime}(\mathrm{G} ; \mathrm{x})\right|_{\mathrm{x}=1}=\sum_{\mathrm{k}=1}^{\delta_{\mathrm{n}}} \mathrm{k} \mathrm{C}_{\mathrm{n}}(\mathrm{G}, \mathrm{k})$

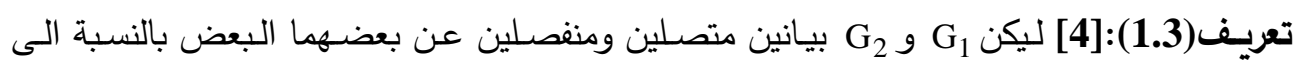
الرؤوس، وليكن

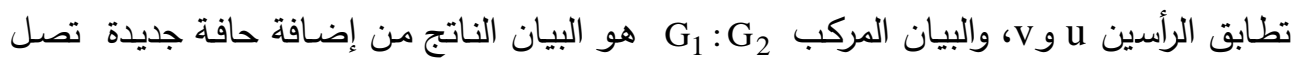
الرأسين u و الرسين v

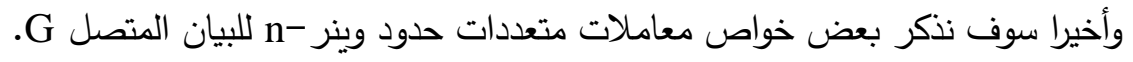

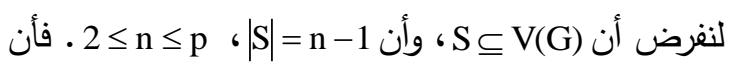

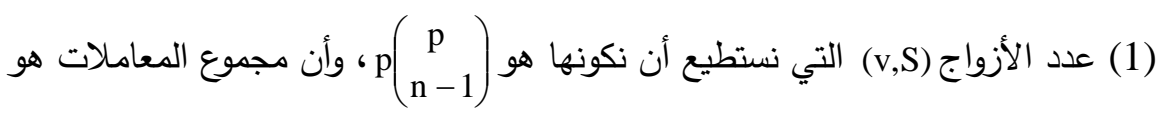

$$
\begin{aligned}
& \sum_{i=1}^{\delta_{n}} C_{n}(G, i)=(p-n+1)\left(\begin{array}{c}
p \\
n-1
\end{array}\right)=p\left(\begin{array}{l}
p-1 \\
n-1
\end{array}\right) \\
& C_{n}(G, 0)=p\left(\begin{array}{l}
p-1 \\
n-2
\end{array}\right)
\end{aligned}
$$

(3) المأخوذة الآتية تعين لنا قيمة المعامل Ce (G,1) لكل بيان متصل. 


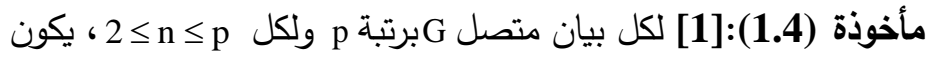

$\mathrm{C}_{\mathrm{n}}(\mathrm{G}, 1)=\mathrm{p}\left(\begin{array}{l}\mathrm{p}-1 \\ \mathrm{n}-1\end{array}\right)-\sum_{\forall \mathrm{v} \in \mathrm{V}}\left(\begin{array}{c}\mathrm{p}-1-\mathrm{deg}_{\mathrm{G}} \mathrm{v} \\ \mathrm{n}-1\end{array}\right) . \quad \#$

2. n متعددة حدود وينر - n لاتصال بيانين:

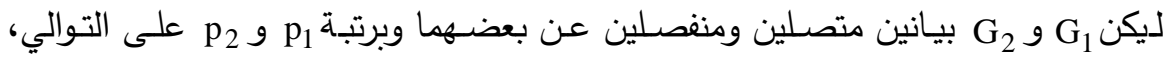

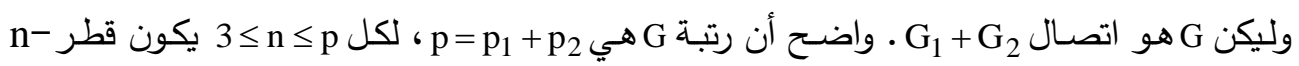

للبيان G لا يزيد على 2.

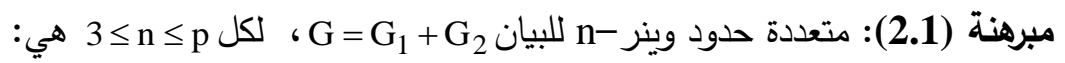

$\mathrm{W}_{\mathrm{n}}(\mathrm{G} ; \mathrm{x})=\sum_{\mathrm{k}=0}^{2} \mathrm{C}_{\mathrm{n}}(\mathrm{G}, \mathrm{k}) \mathrm{x}^{\mathrm{k}}$

إذ أن: - n - n

$C_{n}(G, 0)=p\left(\begin{array}{l}p-1 \\ n-2\end{array}\right)$

$\mathrm{C}_{\mathrm{n}}(\mathrm{G}, 1)=\mathrm{p}\left(\begin{array}{l}\mathrm{p}-1 \\ \mathrm{n}-1\end{array}\right)-\sum_{\mathrm{v} \in \mathrm{V}\left(\mathrm{G}_{1}\right)}\left(\begin{array}{c}\mathrm{p}_{1}-1-\operatorname{deg}_{\mathrm{G}_{1}} \mathrm{v} \\ \mathrm{n}-1\end{array}\right)-\sum_{\mathrm{v} \in \mathrm{V}\left(\mathrm{G}_{2}\right)}\left(\begin{array}{c}\mathrm{p}_{2}-1-\operatorname{deg}_{\mathrm{G}_{2}} \mathrm{v} \\ \mathrm{n}-1\end{array}\right)$

$\mathrm{C}_{\mathrm{n}}(\mathrm{G}, 2)=\sum_{\mathrm{v} \in \mathrm{V}\left(\mathrm{G}_{1}\right)}\left(\begin{array}{c}\mathrm{p}_{1}-1-\operatorname{deg}_{\mathrm{G}_{1}} \mathrm{v} \\ \mathrm{n}-1\end{array}\right)+\sum_{\mathrm{v} \in \mathrm{V}\left(\mathrm{G}_{2}\right)}\left(\begin{array}{c}\mathrm{p}_{2}-1-\operatorname{deg}_{\mathrm{G}_{2}} \mathrm{v} \\ \mathrm{n}-1\end{array}\right)$

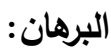

من العلاقة (1.1) نحصل على ما يأتي

$\mathrm{C}_{\mathrm{n}}(\mathrm{G}, 0)=\mathrm{p}\left(\begin{array}{l}\mathrm{p}-1 \\ \mathrm{n}-2\end{array}\right)$

ومن المأخوذة (1.4) ، فأن

$C_{n}(G, 1)=p\left(\begin{array}{l}p-1 \\ n-1\end{array}\right)-\sum_{v \in V(G)}\left(\begin{array}{c}p-1-\operatorname{deg}_{G} v \\ n-1\end{array}\right)$

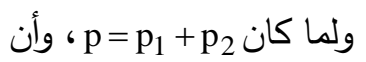

$\operatorname{deg}_{\mathrm{G}} \mathrm{v}= \begin{cases}\mathrm{p}_{2}+\operatorname{deg}_{\mathrm{G}_{1}} \mathrm{v} ; & \text { if } \mathrm{v} \in \mathrm{V}\left(\mathrm{G}_{1}\right) \\ \mathrm{p}_{1}+\operatorname{deg}_{\mathrm{G}_{2}} \mathrm{v} ; & \text { if } \mathrm{v} \in \mathrm{V}\left(\mathrm{G}_{2}\right)\end{cases}$

ولذلك فأن C تكون كما هي مذكورة في نص العبارة 
ولما كان قطر -n للبيان G لا يزيد على 2 ، فأن

$$
\begin{aligned}
& C_{n}(G, 1)+C_{n}(G, 2)=p\left(\begin{array}{l}
p-1 \\
n-1
\end{array}\right)
\end{aligned}
$$

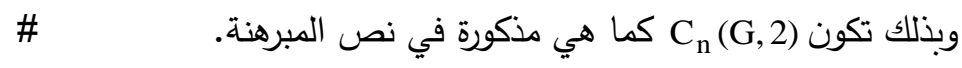

نتيجة (2.2): دليل وينر - لاتصال بيانين منفصلين و $\mathrm{G}_{1}$ و هو

$\mathrm{W}_{\mathrm{n}}\left(\mathrm{G}_{1}+\mathrm{G}_{2}\right)=\mathrm{p}\left(\begin{array}{l}\mathrm{p}-1 \\ \mathrm{n}-1\end{array}\right)+\sum_{\mathrm{v} \in \mathrm{V}\left(\mathrm{G}_{1}\right)}\left(\begin{array}{c}\mathrm{p}_{1}-1-\operatorname{deg}_{\mathrm{G}_{1}} \mathrm{v} \\ \mathrm{n}-1\end{array}\right)+\sum_{\mathrm{v} \in \mathrm{V}\left(\mathrm{G}_{2}\right)}\left(\begin{array}{c}\mathrm{p}_{2}-1-\operatorname{deg}_{\mathrm{G}_{2}} \mathrm{v} \\ \mathrm{n}-1\end{array}\right)$.

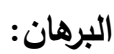

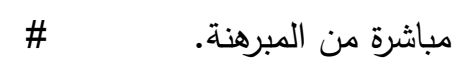

3. متعددة حدود وينر - لبيان مركب من بيانين تامين:

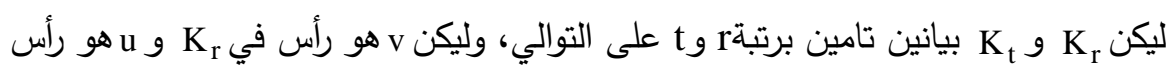

في

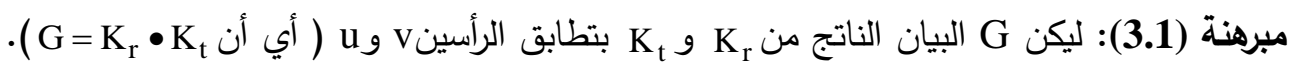

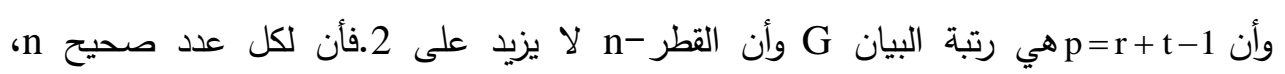
$3 \leq n \leq p$

$\mathrm{W}_{\mathrm{n}}(\mathrm{G} ; \mathrm{x})=\sum_{\mathrm{k}=0}^{2} \mathrm{C}_{\mathrm{n}}(\mathrm{G}, \mathrm{k}) \mathrm{x}^{\mathrm{k}}$

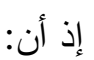

$$
\begin{aligned}
& C_{n}(G, 0)=p\left(\begin{array}{l}
p-1 \\
n-2
\end{array}\right) \\
& C_{n}(G, 1)=p\left(\begin{array}{l}
p-1 \\
n-1
\end{array}\right)-(r-1)\left(\begin{array}{l}
t-1 \\
n-1
\end{array}\right)-(t-1)\left(\begin{array}{l}
\mathrm{r}-1 \\
\mathrm{n}-1
\end{array}\right) \\
& C_{n}(G, 2)=(r-1)\left(\begin{array}{l}
\mathrm{t}-1 \\
n-1
\end{array}\right)+(t-1)\left(\begin{array}{l}
\mathrm{r}-1 \\
\mathrm{n}-1
\end{array}\right)
\end{aligned}
$$

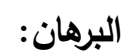

ليكن w هو الرأس الناتج من تطابق الرأسين و u فيG.

فأنه من العلاقة (1.1)، يكون لـ ليكن

$$
C_{n}(G, 0)=p\left(\begin{array}{l}
p-1 \\
n-2
\end{array}\right)
$$

ومن المأخوذة (1.4)، فأن 


$$
\begin{aligned}
C_{n}(G, 1) & =p\left(\begin{array}{l}
p-1 \\
n-1
\end{array}\right)-\sum_{y \in V(G)}\left(\begin{array}{c}
p-1-\operatorname{deg}_{G} y \\
n-1
\end{array}\right) \\
& =p\left(\begin{array}{l}
p-1 \\
n-1
\end{array}\right)-(r-1)\left(\begin{array}{l}
t-1 \\
n-1
\end{array}\right)-(t-1)\left(\begin{array}{l}
r-1 \\
n-1
\end{array}\right)
\end{aligned}
$$

وذلك لأن

$$
\operatorname{deg}_{G} y= \begin{cases}r-1 ; & \text { when } y \in V\left(K_{r}\right)-\{w\} \\ t-1 ; & \text { when } y \in V\left(K_{t}\right)-\{w\} \\ r+t-2 ; & \text { when } y=w\end{cases}
$$

$$
\begin{aligned}
C_{n}(G, 2) & =\sum_{y \in V(G)}\left(\begin{array}{c}
p-1-\operatorname{deg}_{G} y \\
n-1
\end{array}\right) \\
& =(r-1)\left(\begin{array}{l}
t-1 \\
n-1
\end{array}\right)+(t-1)\left(\begin{array}{l}
r-1 \\
n-1
\end{array}\right) \quad \#
\end{aligned}
$$

وبما أن قطر -n للبيان G هو 2 فأن

\section{نتيجة (3.2) :}

$$
\begin{aligned}
& \mathrm{W}_{\mathrm{n}}\left(\mathrm{K}_{\mathrm{r}} \bullet \mathrm{K}_{\mathrm{r}} ; \mathrm{x}\right)=(2 \mathrm{r}-1)\left(\begin{array}{c}
2 \mathrm{r}-2 \\
\mathrm{n}-2
\end{array}\right)+\left[(2 \mathrm{r}-1)\left(\begin{array}{c}
2 \mathrm{r}-2 \\
\mathrm{n}-1
\end{array}\right)-2(\mathrm{r}-1)\left(\begin{array}{l}
\mathrm{r}-1 \\
\mathrm{n}-1
\end{array}\right)\right] \mathrm{x} \\
& +2(\mathrm{r}-1)\left(\begin{array}{l}
\mathrm{r}-1 \\
\mathrm{n}-1
\end{array}\right) \mathrm{x}^{2} ; \quad 3 \leq \mathrm{n} \leq 2 \mathrm{r}-1
\end{aligned}
$$

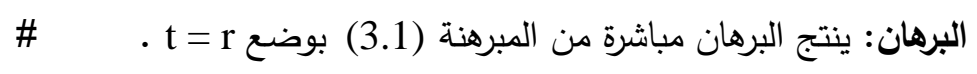

$$
\begin{aligned}
& \mathrm{W}_{\mathrm{n}}\left(\mathrm{K}_{\mathrm{r}} \bullet \mathrm{K}_{\mathrm{t}}\right)=\mathrm{p}\left(\begin{array}{l}
\mathrm{p}-1 \\
\mathrm{n}-1
\end{array}\right)+(\mathrm{r}-1)\left(\begin{array}{l}
\mathrm{t}-1 \\
\mathrm{n}-1
\end{array}\right)+(\mathrm{t}-1)\left(\begin{array}{l}
\mathrm{r}-1 \\
\mathrm{n}-1
\end{array}\right) \text {. } \\
& \text { نتيجة (3.3): دليل وينر -n للبيان }
\end{aligned}
$$

$$
\begin{aligned}
& \text { إذ أن }
\end{aligned}
$$

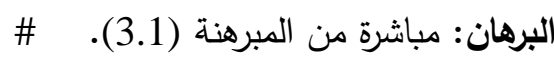

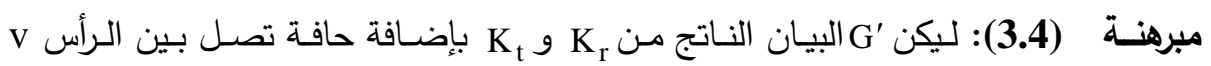

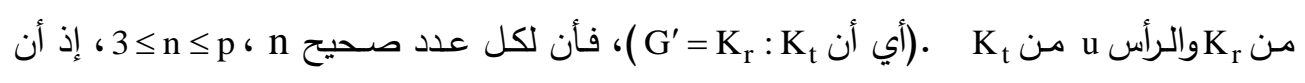
• يكون p=r+t

$$
\mathrm{W}_{\mathrm{n}}\left(\mathrm{G}^{\prime} ; \mathrm{x}\right)=\sum_{\mathrm{k}=0}^{3} \mathrm{C}_{\mathrm{n}}\left(\mathrm{G}^{\prime}, \mathrm{k}\right) \mathrm{x}^{\mathrm{k}}
$$


إذ أن

$C_{n}\left(G^{\prime}, 0\right)=p\left(\begin{array}{l}p-1 \\ n-2\end{array}\right)$

$\mathrm{C}_{\mathrm{n}}\left(\mathrm{G}^{\prime}, 1\right)=\mathrm{p}\left(\begin{array}{l}\mathrm{p}-1 \\ \mathrm{n}-1\end{array}\right)-(\mathrm{rt}-\mathrm{n}+1)\left\{\frac{1}{\mathrm{t}}\left(\begin{array}{c}\mathrm{t} \\ \mathrm{n}-1\end{array}\right)+\frac{1}{\mathrm{r}}\left(\begin{array}{c}\mathrm{r} \\ \mathrm{n}-1\end{array}\right)\right\}$

$\mathrm{C}_{\mathrm{n}}\left(\mathrm{G}^{\prime}, 2\right)=\left(\mathrm{r}-2+\frac{\mathrm{t}}{\mathrm{n}-1}\right)\left(\begin{array}{l}\mathrm{t}-1 \\ \mathrm{n}-2\end{array}\right)+\left(\mathrm{t}-2+\frac{\mathrm{r}}{\mathrm{n}-1}\right)\left(\begin{array}{l}\mathrm{r}-1 \\ \mathrm{n}-2\end{array}\right)$

$C_{n}\left(G^{\prime}, 3\right)=(r-1)\left(\begin{array}{l}t-1 \\ n-1\end{array}\right)+(t-1)\left(\begin{array}{l}r-1 \\ n-1\end{array}\right)$

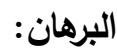

واضنح أن

$C_{n}\left(G^{\prime}, 0\right)=p\left(\begin{array}{l}p-1 \\ n-2\end{array}\right)$

ومن المأخوذة (1.4)، فأن

$\mathrm{C}_{\mathrm{n}}\left(\mathrm{G}^{\prime}, 1\right)=\mathrm{p}\left(\begin{array}{l}\mathrm{p}-1 \\ \mathrm{n}-1\end{array}\right)-\sum_{\mathrm{y} \in \mathrm{V}\left(\mathrm{G}^{\prime}\right)}\left(\begin{array}{c}\mathrm{p}-1-\mathrm{deg}_{\mathrm{G}^{\prime}} \mathrm{y} \\ \mathrm{n}-1\end{array}\right)$

$=p\left(\begin{array}{l}\mathrm{p}-1 \\ \mathrm{n}-1\end{array}\right)-\left\{(\mathrm{r}-1)\left(\begin{array}{c}\mathrm{t} \\ \mathrm{n}-1\end{array}\right)+\left(\begin{array}{l}\mathrm{t}-1 \\ \mathrm{n}-1\end{array}\right)+\left(\begin{array}{l}\mathrm{r}-1 \\ \mathrm{n}-1\end{array}\right)+(\mathrm{t}-1)\left(\begin{array}{c}\mathrm{r} \\ \mathrm{n}-1\end{array}\right)\right\}$ $=\mathrm{p}\left(\begin{array}{l}\mathrm{p}-1 \\ \mathrm{n}-1\end{array}\right)-(\mathrm{rt}-\mathrm{n}+1)\left\{\frac{1}{\mathrm{t}}\left(\begin{array}{c}\mathrm{t} \\ \mathrm{n}-1\end{array}\right)+\frac{1}{\mathrm{r}}\left(\begin{array}{c}\mathrm{r} \\ \mathrm{n}-1\end{array}\right)\right\}$

ولأجل أن نجد

$\mathrm{d}_{\mathrm{n}}(\mathrm{y}, \mathrm{S})=2, \mathrm{y} \in \mathrm{V}\left(\mathrm{G}^{\prime}\right), \mathrm{S} \subseteq \mathrm{V}\left(\mathrm{G}^{\prime}\right),|\mathrm{S}|=\mathrm{n}-1$

فيجب أن يكون لدينا الحالات الآتية:

1) $\mathrm{y}=\mathrm{u} ; \quad \mathrm{S} \subseteq \mathrm{V}\left(\mathrm{K}_{\mathrm{r}}\right)-\{\mathrm{v}\}$

2) $\mathrm{y}=\mathrm{v} ; \quad \mathrm{S} \subseteq \mathrm{V}\left(\mathrm{K}_{\mathrm{t}}\right)-\{\mathrm{u}\}$

3) $\mathrm{y} \in \mathrm{V}\left(\mathrm{K}_{\mathrm{t}}\right)-\{\mathrm{u}\} ; \quad \mathrm{S}=\{\mathrm{v}\} \cup \mathrm{T} ; \phi \neq \mathrm{T} \subseteq \mathrm{V}\left(\mathrm{K}_{\mathrm{r}}\right)-\{\mathrm{v}\}$

4) $\mathrm{y} \in \mathrm{V}\left(\mathrm{K}_{\mathrm{r}}\right)-\{\mathrm{v}\} ; \quad \mathrm{S}=\{\mathrm{u}\} \cup \mathrm{T} ; \phi \neq \mathrm{T} \subseteq \mathrm{V}\left(\mathrm{K}_{\mathrm{t}}\right)-\{\mathrm{u}\}$

$\mathrm{C}_{\mathrm{n}}\left(\mathrm{G}^{\prime}, 2\right)=\left(\begin{array}{l}\mathrm{r}-1 \\ \mathrm{n}-1\end{array}\right)+\left(\begin{array}{l}\mathrm{t}-1 \\ \mathrm{n}-1\end{array}\right)+(\mathrm{t}-1)\left(\begin{array}{l}\mathrm{r}-1 \\ \mathrm{n}-2\end{array}\right)+(\mathrm{r}-1)\left(\begin{array}{l}\mathrm{t}-1 \\ \mathrm{n}-2\end{array}\right)$

وعليه فأن عدد هكذا أزواج (y,S) يكون

والذي يؤدي بالتبسيط إلى الصيغة المعطاة في نص المبرهنة .

أما لإيجاد C C 
$\mathrm{d}_{\mathrm{n}}(\mathrm{y}, \mathrm{S})=3, \mathrm{y} \in \mathrm{V}\left(\mathrm{G}^{\prime}\right), \mathrm{S} \subseteq \mathrm{V}\left(\mathrm{G}^{\prime}\right),|\mathrm{S}|=\mathrm{n}-1$

1) $\mathrm{y} \in \mathrm{V}\left(\mathrm{K}_{\mathrm{r}}\right)-\{\mathrm{v}\} ; \quad \mathrm{S} \subseteq \mathrm{V}\left(\mathrm{K}_{\mathrm{t}}\right)-\{\mathrm{u}\}$

$2 \mathrm{y} \in \mathrm{V}\left(\mathrm{K}_{\mathrm{t}}\right)-\{\mathrm{u}\} ; \quad \mathrm{S} \subseteq \mathrm{V}\left(\mathrm{K}_{\mathrm{r}}\right)-\{\mathrm{v}\}$

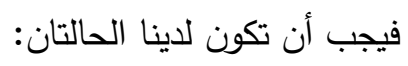

وعليه فأن عدد هكذا أزواج ( y, S ) يكون

$C_{n}\left(G^{\prime}, 3\right)=(r-1)\left(\begin{array}{l}t-1 \\ n-1\end{array}\right)+(t-1)\left(\begin{array}{l}r-1 \\ n-1\end{array}\right)$

\#

يكون G' $^{\prime}=K_{r}: K_{t}$ - للبيان ملاحظة أن القطر

$\mathrm{n}-1<\max \{\mathrm{r}, \mathrm{t}\} ;$ when $\delta_{\mathrm{n}}=3$

$\mathrm{n}-1 \geq \max \{\mathrm{r}, \mathrm{t}\} ; \quad$ when $\delta_{\mathrm{n}}=2,1$

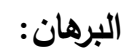

عند اشتقاق متعددة حدود وينر-n للبيان G'=Kr

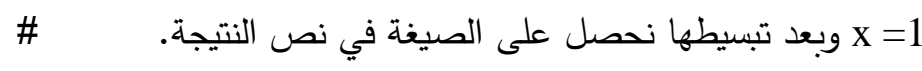

4 4. متعددة حدود وينر - لبيان مركب من نجمتين:

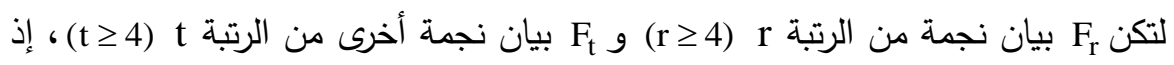

$\mathrm{V}\left(\mathrm{F}_{\mathrm{r}}\right)=\left\{\mathrm{c}_{1}, \mathrm{v}_{1}, \mathrm{v}_{2}, \ldots, \mathrm{v}_{\mathrm{r}-1}\right\}$

$\mathrm{V}\left(\mathrm{F}_{\mathrm{t}}\right)=\left\{\mathrm{c}_{2}, \mathrm{u}_{1}, \mathrm{u}_{2}, \ldots, \mathrm{u}_{\mathrm{t}-1}\right\}$

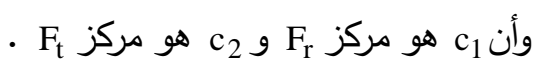

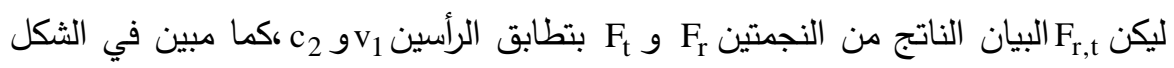




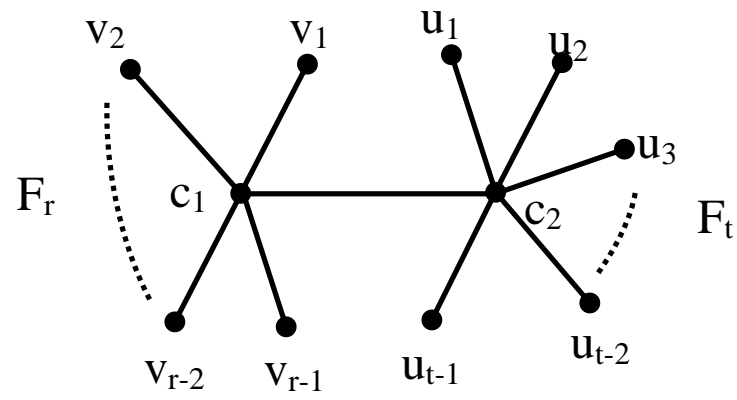

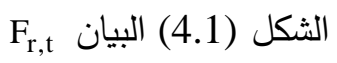

مبرهنة (4.1): متعددة حدود وينر -n للبيان Fي

$\mathrm{W}_{\mathrm{n}}\left(\mathrm{F}_{\mathrm{r}, \mathrm{t}} ; \mathrm{x}\right)=\sum_{\mathrm{k}=0}^{3} \mathrm{C}_{\mathrm{n}}\left(\mathrm{F}_{\mathrm{r}, \mathrm{t}}, \mathrm{k}\right) \mathrm{x}^{\mathrm{k}}$

$\mathrm{C}_{\mathrm{n}}\left(\mathrm{F}_{\mathrm{r}, \mathrm{t}}, 0\right)=\mathrm{p}\left(\begin{array}{l}\mathrm{p}-1 \\ \mathrm{n}-2\end{array}\right)$

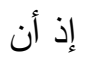

$\mathrm{C}_{\mathrm{n}}\left(\mathrm{F}_{\mathrm{r}, \mathrm{t}}, 1\right)=\mathrm{p}\left(\begin{array}{l}\mathrm{p}-1 \\ \mathrm{n}-1\end{array}\right)-\left\{(\mathrm{p}-2)\left(\begin{array}{l}\mathrm{p}-2 \\ \mathrm{n}-1\end{array}\right)+\left(\begin{array}{l}\mathrm{t}-1 \\ \mathrm{n}-1\end{array}\right)+\left(\begin{array}{l}\mathrm{r}-2 \\ \mathrm{n}-1\end{array}\right)\right\}$

$\mathrm{C}_{\mathrm{n}}\left(\mathrm{F}_{\mathrm{r}, \mathrm{t}}, 2\right)=(\mathrm{p}-2)\left(\begin{array}{l}\mathrm{p}-2 \\ \mathrm{n}-1\end{array}\right)-(\mathrm{r}-3)\left(\begin{array}{l}\mathrm{t}-1 \\ \mathrm{n}-1\end{array}\right)-(\mathrm{t}-2)\left(\begin{array}{l}\mathrm{r}-2 \\ \mathrm{n}-1\end{array}\right)$

$\mathrm{C}_{\mathrm{n}}\left(\mathrm{F}_{\mathrm{r}, \mathrm{t}}, 3\right)=(\mathrm{r}-2)\left(\begin{array}{l}\mathrm{t}-1 \\ \mathrm{n}-1\end{array}\right)+(\mathrm{t}-1)\left(\begin{array}{l}\mathrm{r}-2 \\ \mathrm{n}-1\end{array}\right)$

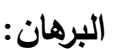

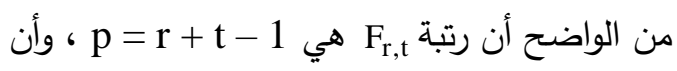

$\mathrm{n}<\max \{\mathrm{r}, \mathrm{t}+1\} ;$ when $\delta_{\mathrm{n}}=3$

$\mathrm{n} \geq \max \{\mathrm{r}, \mathrm{t}+1\} ; \quad$ when $\delta_{\mathrm{n}}=2,1$

$\mathrm{C}_{\mathrm{n}}\left(\mathrm{F}_{\mathrm{r}, \mathrm{t}}, 0\right)=\mathrm{p}\left(\begin{array}{l}\mathrm{p}-1 \\ \mathrm{n}-2\end{array}\right)$

من العلاقة (1.1) نحصل على ما يأتي

ومن المأخوذة (1.2) فأن 


$$
\begin{aligned}
& \mathrm{C}_{\mathrm{n}}\left(\mathrm{F}_{\mathrm{r}, \mathrm{t}}, 1\right)=\mathrm{p}\left(\begin{array}{l}
\mathrm{p}-1 \\
\mathrm{n}-1
\end{array}\right)-\sum_{\mathrm{y} \in \mathrm{V}\left(\mathrm{F}_{\mathrm{r}, \mathrm{t}}\right)}\left(\begin{array}{c}
\mathrm{p}-1-\operatorname{deg}_{\mathrm{F}_{\mathrm{r}, \mathrm{t}}} \mathrm{y} \\
\mathrm{n}-1
\end{array}\right) \\
& =p\left(\begin{array}{l}
\mathrm{p}-1 \\
\mathrm{n}-1
\end{array}\right)-\left\{(\mathrm{p}-2)\left(\begin{array}{l}
\mathrm{p}-2 \\
\mathrm{n}-1
\end{array}\right)+\left(\begin{array}{l}
\mathrm{t}-1 \\
\mathrm{n}-1
\end{array}\right)+\left(\begin{array}{l}
\mathrm{r}-2 \\
\mathrm{n}-1
\end{array}\right)\right\}
\end{aligned}
$$

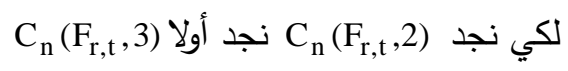

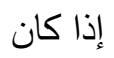$$
\mathrm{d}_{\mathrm{n}}(\mathrm{y}, \mathrm{S})=3, \mathrm{y} \in \mathrm{V}\left(\mathrm{F}_{\mathrm{r}, \mathrm{t}}\right), \mathrm{S} \subseteq \mathrm{V}\left(\mathrm{F}_{\mathrm{r}, \mathrm{t}}\right), \quad|\mathrm{S}|=\mathrm{n}-1
$$

فأنه توجد لدينا حالتان فقط وهما

1) $\mathrm{y} \in \mathrm{V}\left(\mathrm{F}_{\mathrm{r}}\right)-\left\{\mathrm{c}_{1}, v_{1}\right\} ; \quad \mathrm{S} \subseteq \mathrm{V}\left(\mathrm{F}_{\mathrm{t}}\right)-\left\{\mathrm{c}_{2}\right\}$

2) $\mathrm{y} \in \mathrm{V}\left(\mathrm{F}_{\mathrm{t}}\right)-\left\{\mathrm{c}_{2}\right\} ; \quad \mathrm{S} \subseteq \mathrm{V}\left(\mathrm{F}_{\mathrm{r}}\right)-\left\{\mathrm{c}_{1}, v_{1}\right\}$

$\mathrm{C}_{\mathrm{n}}\left(\mathrm{F}_{\mathrm{r}, \mathrm{t}}, 3\right)=(\mathrm{r}-2)\left(\begin{array}{l}\mathrm{t}-1 \\ \mathrm{n}-1\end{array}\right)+(\mathrm{t}-1)\left(\begin{array}{l}\mathrm{r}-2 \\ \mathrm{n}-1\end{array}\right)$

ومنهما نحصل على ما يأتي

أما C

$\mathrm{C}_{\mathrm{n}}\left(\mathrm{F}_{\mathrm{r}, \mathrm{t}}, 2\right)+\mathrm{C}_{\mathrm{n}}\left(\mathrm{F}_{\mathrm{r}, \mathrm{t}}, 3\right)=\sum_{\mathrm{y} \in \mathrm{V}\left(\mathrm{F}_{\mathrm{r}, \mathrm{t}}\right)}\left(\begin{array}{c}\mathrm{p}-1-\operatorname{deg}_{\mathrm{F}_{\mathrm{r}, \mathrm{t}}} \mathrm{y} \\ \mathrm{n}-1\end{array}\right)$

نتيجة (4.2): دليل وينر -n للبيان المركب F, Fو

$\mathrm{W}_{\mathrm{n}}\left(\mathrm{F}_{\mathrm{r}, \mathrm{t}}\right)=\mathrm{p}\left(\begin{array}{l}\mathrm{p}-1 \\ \mathrm{n}-1\end{array}\right)+(\mathrm{p}-2)\left(\begin{array}{l}\mathrm{p}-2 \\ \mathrm{n}-1\end{array}\right)+\mathrm{t}\left(\begin{array}{l}\mathrm{r}-2 \\ \mathrm{n}-1\end{array}\right)+(\mathrm{r}-1)\left(\begin{array}{l}\mathrm{t}-1 \\ \mathrm{n}-1\end{array}\right)$.

\#

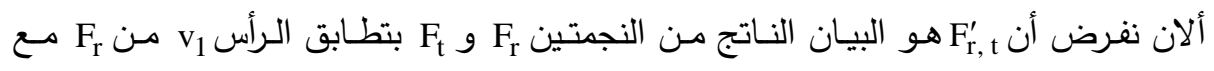

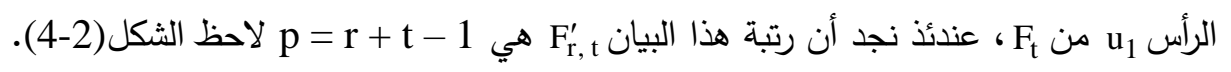




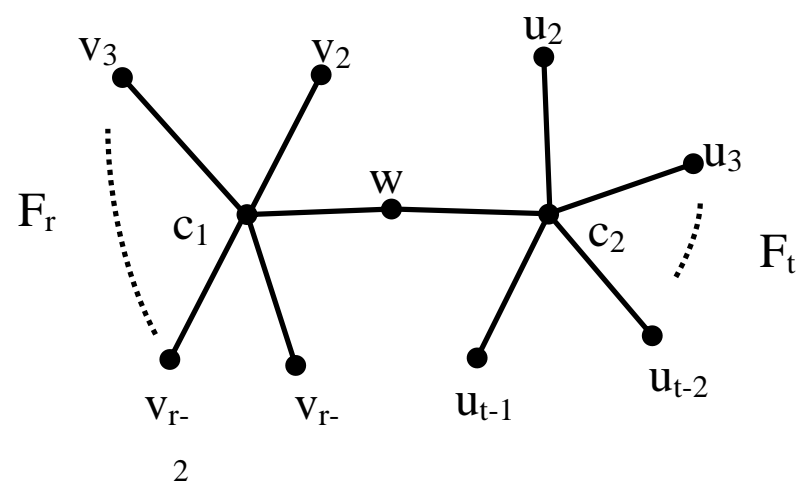

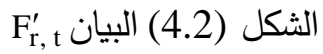

المبرهنة (4.3): متعددة حدود وينر n- للبيان Fي

$\mathrm{W}_{\mathrm{n}}\left(\mathrm{F}_{\mathrm{r}, \mathrm{t}}^{\prime} ; \mathrm{x}\right)=\sum_{\mathrm{k}=0}^{4} \mathrm{C}_{\mathrm{n}}\left(\mathrm{F}_{\mathrm{r}, \mathrm{t}}^{\prime}, \mathrm{k}\right) \mathrm{x}^{\mathrm{k}}$

$\mathrm{C}_{\mathrm{n}}\left(\mathrm{F}_{\mathrm{r}, \mathrm{t}}^{\prime}, 0\right)=\mathrm{p}\left(\begin{array}{l}\mathrm{p}-1 \\ \mathrm{n}-2\end{array}\right)$

$\mathrm{C}_{\mathrm{n}}\left(\mathrm{F}_{\mathrm{r}, \mathrm{t}}^{\prime}, 1\right)=\mathrm{p}\left(\begin{array}{l}\mathrm{p}-1 \\ \mathrm{n}-1\end{array}\right)-\left\{\left(\mathrm{p}-2-\frac{\mathrm{n}-1}{\mathrm{p}-2}\right)\left(\begin{array}{l}\mathrm{p}-2 \\ \mathrm{n}-1\end{array}\right)+\left(\begin{array}{l}\mathrm{t}-1 \\ \mathrm{n}-1\end{array}\right)+\left(\begin{array}{l}\mathrm{r}-1 \\ \mathrm{n}-1\end{array}\right)\right\}$

$C_{n}\left(F_{r, t}^{\prime}, 2\right)=\left(p-2-\frac{n-1}{p-2}\right)\left(\begin{array}{l}p-2 \\ n-1\end{array}\right)-\left(t-2-\frac{n-1}{r-1}\right)\left(\begin{array}{l}r-1 \\ n-1\end{array}\right)-\left(r-2-\frac{n-1}{t-1}\right)\left(\begin{array}{l}t-1 \\ n-1\end{array}\right)$

$C_{n}\left(F_{r, t}^{\prime}, 3\right)=\left(r-3+\frac{t-1}{n-1}\right)\left(\begin{array}{l}t-2 \\ n-2\end{array}\right)+\left(t-3+\frac{r-1}{n-1}\right)\left(\begin{array}{l}r-2 \\ n-2\end{array}\right)$

$\mathrm{C}_{\mathrm{n}}\left(\mathrm{F}_{\mathrm{r}, \mathrm{t}}^{\prime}, 4\right)=(\mathrm{r}-2)\left(\begin{array}{l}\mathrm{t}-2 \\ \mathrm{n}-1\end{array}\right)+(\mathrm{t}-2)\left(\begin{array}{l}\mathrm{r}-2 \\ \mathrm{n}-1\end{array}\right)$

إذ أن

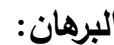

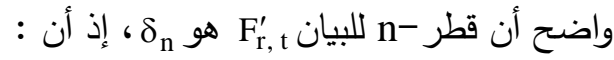

$\mathrm{n}<\max \{\mathrm{r}, \mathrm{t}\} ;$ when $\delta_{\mathrm{n}}=4$

$\mathrm{n} \geq \max \{\mathrm{r}, \mathrm{t}\} ; \quad$ when $\delta_{\mathrm{n}}=3,2,1$

$\mathrm{C}_{\mathrm{n}}\left(\mathrm{F}_{\mathrm{r}, \mathrm{t}}^{\prime}, 0\right)=\mathrm{p}\left(\begin{array}{l}\mathrm{p}-1 \\ \mathrm{n}-2\end{array}\right)$ 
ومن المأخوذة (1.4)، فأن

$$
\begin{aligned}
\mathrm{C}_{\mathrm{n}}\left(\mathrm{F}_{\mathrm{r}, \mathrm{t}}^{\prime}, 1\right) & =\mathrm{p}\left(\begin{array}{l}
\mathrm{p}-1 \\
\mathrm{n}-1
\end{array}\right)-\sum_{\mathrm{y} \in \mathrm{V}\left(\mathrm{F}_{\mathrm{r}, \mathrm{t}}^{\prime}\right.}\left(\begin{array}{c}
\mathrm{p}-1-\operatorname{deg}_{\mathrm{F}_{\mathrm{r}, \mathrm{t}}^{\prime} \mathrm{y}} \\
\mathrm{n}-1
\end{array}\right) \\
& =\mathrm{p}\left(\begin{array}{l}
\mathrm{p}-1 \\
\mathrm{n}-1
\end{array}\right)-\left\{(\mathrm{p}-3)\left(\begin{array}{l}
\mathrm{p}-2 \\
\mathrm{n}-1
\end{array}\right)+\left(\begin{array}{l}
\mathrm{p}-3 \\
\mathrm{n}-1
\end{array}\right)+\left(\begin{array}{l}
\mathrm{t}-1 \\
\mathrm{n}-1
\end{array}\right)+\left(\begin{array}{l}
\mathrm{r}-1 \\
\mathrm{n}-1
\end{array}\right)\right\}
\end{aligned}
$$

ويمكن تبسيط المقدار في الطرف الأيمن للحصول على المقدار المعطى في نص المبرهنة .

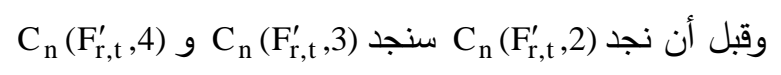

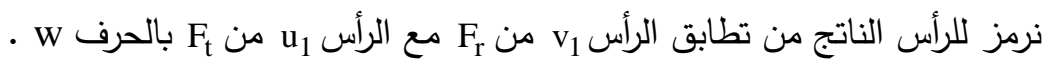

فإذا كان

$\mathrm{d}_{\mathrm{n}}(\mathrm{y}, \mathrm{S})=3, \mathrm{y} \in \mathrm{V}\left(\mathrm{F}_{\mathrm{r}, \mathrm{t}}^{\prime}\right), \mathrm{S} \subseteq \mathrm{V}\left(\mathrm{F}_{\mathrm{r}, \mathrm{t}}^{\prime}\right),|\mathrm{S}|=\mathrm{n}-1$

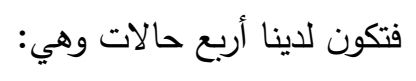

1) $\mathrm{y}=\mathrm{c}_{1} ; \quad \mathrm{S} \subseteq \mathrm{V}\left(\mathrm{F}_{\mathrm{t}}\right)-\left\{\mathrm{w}, \mathrm{c}_{2}\right\}$

2) $\mathrm{y}=\mathrm{c}_{2} ; \quad \mathrm{S} \subseteq \mathrm{V}\left(\mathrm{F}_{\mathrm{r}}\right)-\left\{\mathrm{w}, \mathrm{c}_{1}\right\}$

3) $\mathrm{y} \in \mathrm{V}\left(\mathrm{F}_{\mathrm{r}}\right)-\left\{\mathrm{w}, \mathrm{c}_{1}\right\} ; \quad \mathrm{S}=\left\{\mathrm{c}_{2}\right\} \bigcup \mathrm{T} ; \phi \neq \mathrm{T} \subseteq \mathrm{V}\left(\mathrm{F}_{\mathrm{t}}\right)-\left\{\mathrm{w}, \mathrm{c}_{2}\right\}$

4) $\mathrm{y} \in \mathrm{V}\left(\mathrm{F}_{\mathrm{t}}\right)-\left\{\mathrm{w}, \mathrm{c}_{2}\right\} ; \quad \mathrm{S}=\left\{\mathrm{c}_{1}\right\} \bigcup \mathrm{T} ; \phi \neq \mathrm{T} \subseteq \mathrm{V}\left(\mathrm{F}_{\mathrm{r}}\right)-\left\{\mathrm{w}, \mathrm{c}_{1}\right\}$

$\mathrm{C}_{\mathrm{n}}\left(\mathrm{F}_{\mathrm{r}, \mathrm{t}}^{\prime}, 3\right)=\left(\begin{array}{l}\mathrm{t}-2 \\ \mathrm{n}-1\end{array}\right)+\left(\begin{array}{l}\mathrm{r}-2 \\ \mathrm{n}-1\end{array}\right)+(\mathrm{r}-2)\left(\begin{array}{l}\mathrm{t}-2 \\ \mathrm{n}-2\end{array}\right)+(\mathrm{t}-2)\left(\begin{array}{l}\mathrm{r}-2 \\ \mathrm{n}-2\end{array}\right)$

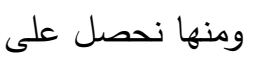

وبعد التبسيط نحصل على الصيغة المذكورة في المبرهنة.

وإذا كان

$\mathrm{d}_{\mathrm{n}}(\mathrm{y}, \mathrm{S})=4, \mathrm{y} \in \mathrm{V}\left(\mathrm{F}_{\mathrm{r}, \mathrm{t}}^{\prime}\right), \mathrm{S} \subseteq \mathrm{V}\left(\mathrm{F}_{\mathrm{r}, \mathrm{t}}^{\prime}\right),|\mathrm{S}|=\mathrm{n}-1$

فيكون لدينا حالتان فقط وهما:

1) $\mathrm{y} \in \mathrm{V}\left(\mathrm{F}_{\mathrm{r}}\right)-\left\{\mathrm{w}, \mathrm{c}_{1}\right\} ; \quad \mathrm{S} \subseteq \mathrm{V}\left(\mathrm{F}_{\mathrm{t}}\right)-\left\{\mathrm{w}, \mathrm{c}_{2}\right\}$

2) $\mathrm{y} \in \mathrm{V}\left(\mathrm{F}_{\mathrm{t}}\right)-\left\{\mathrm{w}, \mathrm{c}_{2}\right\}, \quad \mathrm{S} \subseteq \mathrm{V}\left(\mathrm{F}_{\mathrm{r}}\right)-\left\{\mathrm{w}, \mathrm{c}_{1}\right\}$ 
ومن الحالتين المذكورتين نحصل على ما يأتي:

$$
\mathrm{C}_{\mathrm{n}}\left(\mathrm{F}_{\mathrm{r}, \mathrm{t}}^{\prime}, 4\right)=(\mathrm{r}-2)\left(\begin{array}{l}
\mathrm{t}-2 \\
\mathrm{n}-1
\end{array}\right)+(\mathrm{t}-2)\left(\begin{array}{l}
\mathrm{r}-2 \\
\mathrm{n}-1
\end{array}\right)
$$

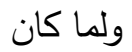

$$
\mathrm{C}_{\mathrm{n}}\left(\mathrm{F}_{\mathrm{r}, \mathrm{t}}^{\prime}, 2\right)+\mathrm{C}_{\mathrm{n}}\left(\mathrm{F}_{\mathrm{r}, \mathrm{t}}^{\prime}, 3\right)+\mathrm{C}_{\mathrm{n}}\left(\mathrm{F}_{\mathrm{r}, \mathrm{t}}^{\prime}, 4\right)=\sum_{\mathrm{y} \in \mathrm{V}\left(\mathrm{F}_{\mathrm{r}, \mathrm{t}}^{\prime}\right)}\left(\begin{array}{c}
\mathrm{p}-1-\operatorname{deg}_{\mathrm{F}_{\mathrm{r}, \mathrm{t}}^{\prime}} \mathrm{y} \\
\mathrm{n}-1
\end{array}\right)
$$

$\mathrm{C}_{\mathrm{n}}\left(\mathrm{F}_{\mathrm{r}, \mathrm{t}}^{\prime}, 2\right)=\left(\mathrm{p}-2-\frac{\mathrm{n}-1}{\mathrm{p}-2}\right)\left(\begin{array}{l}\mathrm{p}-2 \\ \mathrm{n}-1\end{array}\right)+\left(\begin{array}{l}\mathrm{t}-2 \\ \mathrm{n}-2\end{array}\right)+\left(\begin{array}{l}\mathrm{r}-2 \\ \mathrm{n}-2\end{array}\right)-(\mathrm{r}-2)\left(\begin{array}{l}\mathrm{t}-1 \\ \mathrm{n}-1\end{array}\right)-(\mathrm{t}-2)\left(\begin{array}{l}\mathrm{r}-1 \\ \mathrm{n}-1\end{array}\right)$

$$
=\left(\mathrm{p}-2-\frac{\mathrm{n}-1}{\mathrm{p}-2}\right)\left(\begin{array}{l}
\mathrm{p}-2 \\
\mathrm{n}-1
\end{array}\right)-\left(\mathrm{t}-2-\frac{\mathrm{n}-1}{\mathrm{r}-1}\right)\left(\begin{array}{l}
\mathrm{r}-1 \\
\mathrm{n}-1
\end{array}\right)-\left(\mathrm{r}-2-\frac{\mathrm{n}-1}{\mathrm{t}-1}\right)\left(\begin{array}{l}
\mathrm{t}-1 \\
\mathrm{n}-1
\end{array}\right)
$$

\#

نتيجة (4.4): ليل وينر - للبيان المركب $\mathrm{W}_{\mathrm{n}}\left(\mathrm{F}_{\mathrm{r}, \mathrm{t}}^{\prime}\right)=\mathrm{p}\left(\begin{array}{l}\mathrm{p}-1 \\ \mathrm{n}-1\end{array}\right)+\left(\mathrm{p}-2-\frac{\mathrm{n}-1}{\mathrm{p}-2}\right)\left(\begin{array}{l}\mathrm{p}-2 \\ \mathrm{n}-1\end{array}\right)+(\mathrm{r}-1)\left(2-\frac{\mathrm{n}-1}{\mathrm{t}-1}\right)\left(\begin{array}{l}\mathrm{t}-1 \\ \mathrm{n}-1\end{array}\right)$

$$
+(\mathrm{t}-1)\left(2-\frac{\mathrm{n}-1}{\mathrm{r}-1}\right)\left(\begin{array}{c}
\mathrm{r}-1 \\
\mathrm{n}-1
\end{array}\right)
$$

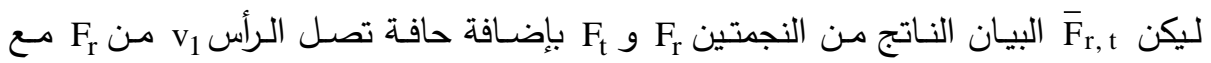

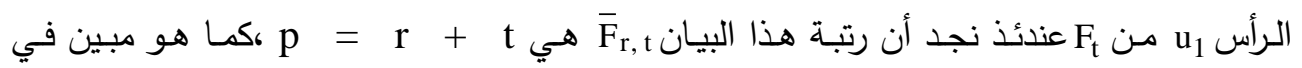

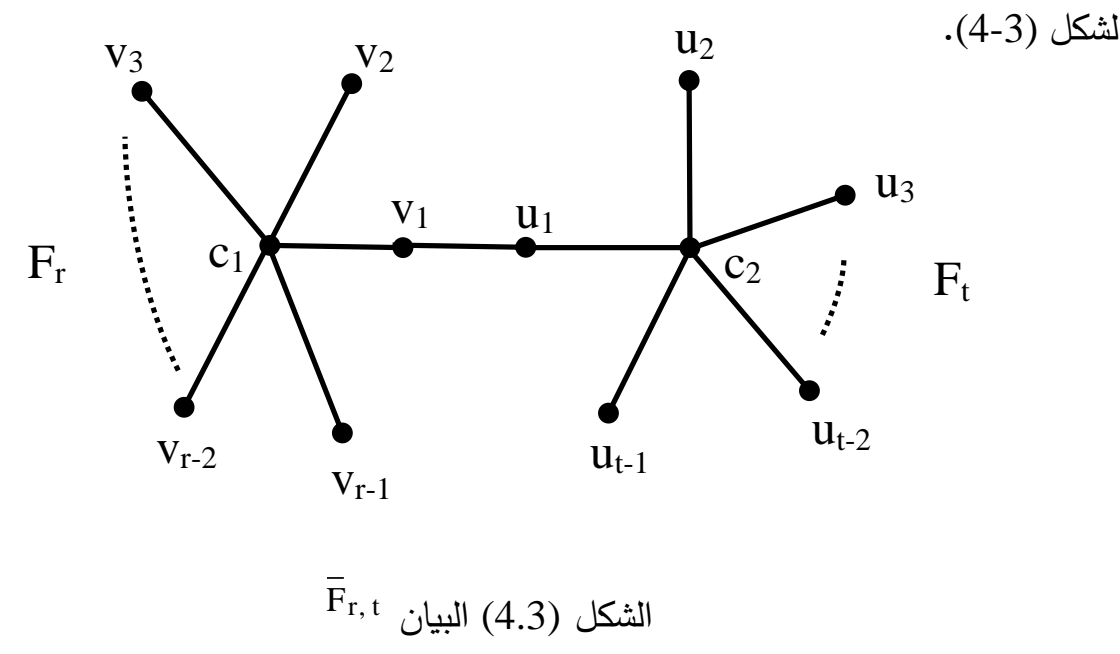


مبرهنة (4.5): متعدة حدود وينر - للبيان

$$
\begin{aligned}
& \mathrm{W}_{\mathrm{n}}\left(\overline{\mathrm{F}}_{\mathrm{r}, \mathrm{t}} ; \mathrm{x}\right)=\sum_{\mathrm{k}=0}^{5} \mathrm{C}_{\mathrm{n}}\left(\overline{\mathrm{F}}_{\mathrm{r}, \mathrm{t}, \mathrm{k}) \mathrm{x}}^{\mathrm{k}}\right. \\
& \mathrm{C}_{\mathrm{n}}\left(\overline{\mathrm{F}}_{\mathrm{r}, \mathrm{t}}, 0\right)=\mathrm{p}\left(\begin{array}{l}
\mathrm{p}-1 \\
\mathrm{n}-2
\end{array}\right) \\
& \mathrm{C}_{\mathrm{n}}\left(\overline{\mathrm{F}}_{\mathrm{r}, \mathrm{t}}, 1\right)=\mathrm{p}\left(\begin{array}{l}
\mathrm{p}-1 \\
\mathrm{n}-1
\end{array}\right)-\left\{\left(\mathrm{p}-2-\frac{2 \mathrm{n}-2}{\mathrm{p}-2}\right)\left(\begin{array}{l}
\mathrm{p}-2 \\
\mathrm{n}-1
\end{array}\right)+\left(\begin{array}{c}
\mathrm{t} \\
\mathrm{n}-1
\end{array}\right)+\left(\begin{array}{c}
\mathrm{r} \\
\mathrm{n}-1
\end{array}\right)\right\} \\
& \mathrm{C}_{\mathrm{n}}\left(\overline{\mathrm{F}}_{\mathrm{r}, \mathrm{t}}, 2\right)=\left(\mathrm{p}-2-\frac{2 \mathrm{n}-2}{\mathrm{p}-2}\right)\left(\begin{array}{l}
\mathrm{p}-2 \\
\mathrm{n}-1
\end{array}\right)-(\mathrm{r}-3)\left(\begin{array}{c}
\mathrm{t} \\
\mathrm{n}-1
\end{array}\right)-(\mathrm{t}-3)\left(\begin{array}{c}
\mathrm{r} \\
\mathrm{n}-1
\end{array}\right) \\
& -\left(2-\frac{\mathrm{n}-1}{\mathrm{r}-1}\right)\left(\begin{array}{l}
\mathrm{r}-1 \\
\mathrm{n}-1
\end{array}\right)-\left(2-\frac{\mathrm{n}-1}{\mathrm{t}-1}\right)\left(\begin{array}{c}
\mathrm{t}-1 \\
\mathrm{n}-1
\end{array}\right) \\
& \mathrm{C}_{\mathrm{n}}\left(\overline{\mathrm{F}}_{\mathrm{r}, \mathrm{t}}, 3\right)=\left(\mathrm{r}-3+\frac{\mathrm{t}}{\mathrm{n}-1}\right)\left(\begin{array}{l}
\mathrm{t}-1 \\
\mathrm{n}-2
\end{array}\right)+\left(\mathrm{t}-3+\frac{\mathrm{r}}{\mathrm{n}-1}\right)\left(\begin{array}{l}
\mathrm{r}-1 \\
\mathrm{n}-2
\end{array}\right) \\
& \mathrm{C}_{\mathrm{n}}\left(\overline{\mathrm{F}}_{\mathrm{r}, \mathrm{t}}, 4\right)=\left(\mathrm{r}-3+\frac{\mathrm{t}-1}{\mathrm{n}-1}\right)\left(\begin{array}{l}
\mathrm{t}-2 \\
\mathrm{n}-2
\end{array}\right)+\left(\mathrm{t}-3+\frac{\mathrm{r}-1}{\mathrm{n}-1}\right)\left(\begin{array}{l}
\mathrm{r}-2 \\
\mathrm{n}-2
\end{array}\right) \\
& C_{n}\left(\bar{F}_{r, t}, 5\right)=(r-2)\left(\begin{array}{l}
t-2 \\
n-1
\end{array}\right)+(t-2)\left(\begin{array}{l}
r-2 \\
n-1
\end{array}\right)
\end{aligned}
$$

إن القطر -n للبيان

$\mathrm{n}<\max \{\mathrm{r}, \mathrm{t}\} ;$ when $\delta_{\mathrm{n}}=5$

$\mathrm{n} \geq \max \{\mathrm{r}, \mathrm{t}\} ;$ when $1 \leq \delta_{\mathrm{n}} \leq 4$

من الواضح أن

$$
\begin{aligned}
\mathrm{C}_{\mathrm{n}}\left(\overline{\mathrm{F}}_{\mathrm{r}, \mathrm{t}}, 0\right) & =\mathrm{p}\left(\begin{array}{l}
\mathrm{p}-1 \\
\mathrm{n}-2
\end{array}\right) \\
\mathrm{C}_{\mathrm{n}}\left(\overline{\mathrm{F}}_{\mathrm{r}, \mathrm{t}}, 1\right) & =\mathrm{p}\left(\begin{array}{l}
\mathrm{p}-1 \\
\mathrm{n}-1
\end{array}\right)-\sum_{\mathrm{y} \in \mathrm{V}\left(\overline{\bar{F}}_{\mathrm{r}, \mathrm{t}}\right)}\left(\begin{array}{c}
\mathrm{p}-1-\operatorname{deg}_{\overline{\mathrm{F}}_{\mathrm{r}, \mathrm{t}}} \mathrm{y} \\
\mathrm{n}-1
\end{array}\right) \\
& =\mathrm{p}\left(\begin{array}{l}
\mathrm{p}-1 \\
\mathrm{n}-1
\end{array}\right)-\left\{(\mathrm{p}-4)\left(\begin{array}{l}
\mathrm{p}-2 \\
\mathrm{n}-1
\end{array}\right)+2\left(\begin{array}{l}
\mathrm{p}-3 \\
\mathrm{n}-1
\end{array}\right)+\left(\begin{array}{c}
\mathrm{r} \\
\mathrm{n}-1
\end{array}\right)+\left(\begin{array}{c}
\mathrm{t} \\
\mathrm{n}-1
\end{array}\right)\right\}
\end{aligned}
$$

وأن 


$$
\begin{aligned}
& =p\left(\begin{array}{l}
\mathrm{p}-1 \\
\mathrm{n}-1
\end{array}\right)-\left\{\left(\mathrm{p}-2-\frac{2 \mathrm{n}-2}{\mathrm{p}-2}\right)\left(\begin{array}{l}
\mathrm{p}-2 \\
\mathrm{n}-1
\end{array}\right)+\left(\begin{array}{c}
\mathrm{t} \\
\mathrm{n}-1
\end{array}\right)+\left(\begin{array}{c}
\mathrm{r} \\
\mathrm{n}-1
\end{array}\right)\right\} \\
& C_{n}\left(\bar{F}_{r, t}, 5\right) \text {, C } C_{n}\left(\bar{F}_{r, t}, 4\right) ، C_{n}\left(\bar{F}_{r, t}, 3\right) \text { آن سوف نجد كلا من }
\end{aligned}
$$

$\mathrm{d}_{\mathrm{n}}(\mathrm{y}, \mathrm{S})=3, \mathrm{y} \in \mathrm{V}\left(\overline{\mathrm{F}}_{\mathrm{r}, \mathrm{t}}\right), \mathrm{S} \subseteq \mathrm{V}\left(\overline{\mathrm{F}}_{\mathrm{r}, \mathrm{t}}\right),|\mathrm{S}|=\mathrm{n}-1$

فأنه لدينا ست حالات وهي

1) $\mathrm{y} \in \mathrm{V}\left(\mathrm{F}_{\mathrm{r}}\right)-\left\{\mathrm{c}_{1}, \mathrm{v}_{1}\right\} ; \quad \mathrm{S}=\left\{\mathrm{u}_{1}\right\} \bigcup \mathrm{T} ; \phi \neq \mathrm{T} \subseteq \mathrm{V}\left(\mathrm{F}_{\mathrm{t}}\right)-\left\{\mathrm{u}_{1}\right\}$

2) $\mathrm{y} \in \mathrm{V}\left(\mathrm{F}_{\mathrm{t}}\right)-\left\{\mathrm{c}_{2}, \mathrm{u}_{1}\right\} ; \quad \mathrm{S}=\left\{\mathrm{v}_{1}\right\} \cup \mathrm{T} ; \phi \neq \mathrm{T} \subseteq \mathrm{V}\left(\mathrm{F}_{\mathrm{r}}\right)-\left\{\mathrm{v}_{1}\right\}$

3) $\mathrm{y}=\mathrm{c}_{1} ; \quad \mathrm{S}=\left\{\mathrm{c}_{2}\right\} \bigcup \mathrm{T} ; \phi \neq \mathrm{T} \subseteq \mathrm{V}\left(\mathrm{F}_{\mathrm{t}}\right)-\left\{\mathrm{c}_{2}, \mathrm{u}_{1}\right\}$

4) $\mathrm{y}=\mathrm{c}_{2} ; \quad \mathrm{S}=\left\{\mathrm{c}_{1}\right\} \bigcup \mathrm{T} ; \phi \neq \mathrm{T} \subseteq \mathrm{V}\left(\mathrm{F}_{\mathrm{r}}\right)-\left\{\mathrm{c}_{1}, \mathrm{v}_{1}\right\}$

5) $\mathrm{y}=\mathrm{v}_{1} ; \quad \mathrm{S} \subseteq \mathrm{V}\left(\mathrm{F}_{\mathrm{t}}\right)-\left\{\mathrm{c}_{2}, \mathrm{u}_{1}\right\}$

6) $\mathrm{y}=\mathrm{u}_{1} ; \quad \mathrm{S} \subseteq \mathrm{V}\left(\mathrm{F}_{\mathrm{r}}\right)-\left\{\mathrm{c}_{1}, \mathrm{v}_{1}\right\}$

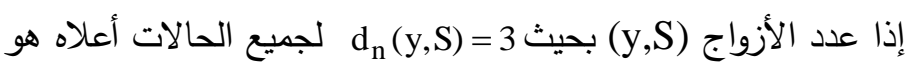

$\mathrm{C}_{\mathrm{n}}\left(\overline{\mathrm{F}}_{\mathrm{r}, \mathrm{t}}, 3\right)=(\mathrm{r}-2)\left(\begin{array}{l}\mathrm{t}-1 \\ \mathrm{n}-2\end{array}\right)+(\mathrm{t}-2)\left(\begin{array}{l}\mathrm{r}-1 \\ \mathrm{n}-2\end{array}\right)+\left(\begin{array}{l}\mathrm{t}-2 \\ \mathrm{n}-2\end{array}\right)+\left(\begin{array}{l}\mathrm{r}-2 \\ \mathrm{n}-2\end{array}\right)+\left(\begin{array}{l}\mathrm{t}-2 \\ \mathrm{n}-1\end{array}\right)+\left(\begin{array}{l}\mathrm{r}-2 \\ \mathrm{n}-1\end{array}\right)$

بعد التبسيط نحصل على Cبالصيغة المذكورة في نص المبرهنة . وإذا كان

$\mathrm{d}_{\mathrm{n}}(\mathrm{y}, \mathrm{S})=4, \mathrm{y} \in \mathrm{V}\left(\overline{\mathrm{F}}_{\mathrm{r}, \mathrm{t}}\right), \mathrm{S} \subseteq \mathrm{V}\left(\overline{\mathrm{F}}_{\mathrm{r}, \mathrm{t}}\right),|\mathrm{S}|=\mathrm{n}-1$

فأن لدينا أربع حالات وهي

1) $\mathrm{y} \in \mathrm{V}\left(\mathrm{F}_{\mathrm{r}}\right)-\left\{\mathrm{c}_{1}, \mathrm{v}_{1}\right\} ; \quad \mathrm{S}=\left\{\mathrm{c}_{2}\right\} \cup \mathrm{T} ; \phi \neq \mathrm{T} \subseteq \mathrm{V}\left(\mathrm{F}_{\mathrm{t}}\right)-\left\{\mathrm{c}_{2}, \mathrm{u}_{1}\right\}$

2) $\mathrm{y} \in \mathrm{V}\left(\mathrm{F}_{\mathrm{t}}\right)-\left\{\mathrm{c}_{2}, \mathrm{u}_{1}\right\} ; \quad \mathrm{S}=\left\{\mathrm{c}_{1}\right\} \bigcup \mathrm{T} ; \phi \neq \mathrm{T} \subseteq \mathrm{V}\left(\mathrm{F}_{\mathrm{r}}\right)-\left\{\mathrm{c}_{1}, \mathrm{v}_{1}\right\}$

3) $\mathrm{y}=\mathrm{c}_{1} ; \quad \mathrm{S} \subseteq \mathrm{V}\left(\mathrm{F}_{\mathrm{t}}\right)-\left\{\mathrm{c}_{2}, \mathrm{u}_{1}\right\}$

4) $\mathrm{y}=\mathrm{c}_{2} ; \quad \mathrm{S} \subseteq \mathrm{V}\left(\mathrm{F}_{\mathrm{r}}\right)-\left\{\mathrm{c}_{1}, \mathrm{v}_{1}\right\}$

إذا

$C_{n}\left(\bar{F}_{r, t}, 4\right)=(r-2)\left(\begin{array}{l}t-2 \\ n-2\end{array}\right)+(t-2)\left(\begin{array}{l}r-2 \\ n-2\end{array}\right)+\left(\begin{array}{l}t-2 \\ n-1\end{array}\right)+\left(\begin{array}{l}r-2 \\ n-1\end{array}\right)$ 
وأيضا بعد التبسيط نحصل على C ${ }_{n}\left(\bar{F}_{r, t}, 4\right)$ وإذا كان

$$
\mathrm{d}_{\mathrm{n}}(\mathrm{y}, \mathrm{S})=5, \mathrm{y} \in \mathrm{V}\left(\overline{\mathrm{F}}_{\mathrm{r}, \mathrm{t}}\right), \mathrm{S} \subseteq \mathrm{V}\left(\overline{\mathrm{F}}_{\mathrm{r}, \mathrm{t}}\right),|\mathrm{S}|=\mathrm{n}-1
$$

فأنه توجد لدينا حالتان هما :

1) $\mathrm{y} \in \mathrm{V}\left(\mathrm{F}_{\mathrm{r}}\right)-\left\{\mathrm{c}_{1}, \mathrm{v}_{1}\right\} ; \quad \mathrm{S} \subseteq \mathrm{V}\left(\mathrm{F}_{\mathrm{t}}\right)-\left\{\mathrm{c}_{2}, \mathrm{u}_{1}\right\}$

2) $\mathrm{y} \in \mathrm{V}\left(\mathrm{F}_{\mathrm{t}}\right)-\left\{\mathrm{c}_{2}, \mathrm{u}_{1}\right\} ; \quad \mathrm{S} \subseteq \mathrm{V}\left(\mathrm{F}_{\mathrm{r}}\right)-\left\{\mathrm{c}_{1}, \mathrm{v}_{1}\right\}$

وبهاتين الحالتين نحصل على $C_{n}\left(\bar{F}_{r, t}, 5\right.$ هي مذكورة في نص المبرهنة. وأخيرا نحصل على $C_{n}\left(\bar{F}_{r, t}, 2\right)$

$\mathrm{C}_{\mathrm{n}}\left(\overline{\mathrm{F}}_{\mathrm{r}, \mathrm{t}}, 2\right)+\sum_{\mathrm{k}=3}^{5} \mathrm{C}_{\mathrm{n}}\left(\overline{\mathrm{F}}_{\mathrm{r}, \mathrm{t}}, \mathrm{k}\right)=\sum_{\mathrm{y} \in \mathrm{V}\left(\mathrm{F}_{\mathrm{r}, \mathrm{t}}\right)}\left(\begin{array}{c}\mathrm{p}-1-\mathrm{deg}_{\overline{\mathrm{F}}_{\mathrm{r}, \mathrm{t}}} \mathrm{y} \\ \mathrm{n}-1\end{array}\right)$

\#

نتيجة (4.6): دليل وينر - للبيان المركب

$\mathrm{W}_{\mathrm{n}}\left(\overline{\mathrm{F}}_{\mathrm{r}, \mathrm{t}}\right)=\mathrm{p}\left(\begin{array}{l}\mathrm{p}-1 \\ \mathrm{n}-1\end{array}\right)+\left(\mathrm{p}-2-\frac{2 \mathrm{n}-2}{\mathrm{p}-2}\right)\left(\begin{array}{l}\mathrm{p}-2 \\ \mathrm{n}-1\end{array}\right)+(\mathrm{r}-1)\left(\begin{array}{c}\mathrm{t} \\ \mathrm{n}-1\end{array}\right)+(\mathrm{t}-1)\left(\begin{array}{c}\mathrm{r} \\ \mathrm{n}-1\end{array}\right)$ $+\left(2 \mathrm{t}-1-\frac{\mathrm{t}(\mathrm{n}-1)}{\mathrm{r}-1}\right)\left(\begin{array}{l}\mathrm{r}-1 \\ \mathrm{n}-1\end{array}\right)+\left(2 \mathrm{r}-1-\frac{\mathrm{r}(\mathrm{n}-1)}{\mathrm{t}-1}\right)\left(\begin{array}{l}\mathrm{t}-1 \\ \mathrm{n}-1\end{array}\right)$

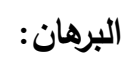

بعد اشتقاق متعددة حدود وينر n- للبيان

ذلك نقوم بإجراء بعض الاختصارات فنحصل على الصيغة المعطاة في النتيجة. ل 


\section{المصادر}

[1] Ali A.M. ; (2005), Wiener Polynomials of Generalized Distance in Graphs, M.SC. Thesis, Mosul University, Mosul.

[2] Buckley F. and Harary F. ; (1990). Distance in Graphs. Addison -Wesley, New York.

[3] Dankelmann P. , Goddard W. , Henning M.A. and Swart H.C. ; (1999).Generalized eccentricity, radius and diameter in graphs. Foundation for Research Development . c 1999 John Wiely \& Sons , Inc. Networks 34: 312-319.

[4] Gutman I. ; (1993). Some properties of the Wiener polynomial ; Graph Theory Notes of New York; XXV .The New York Academy of Sciences 13-17. 